\title{
LSS with angular cross-correlations: Combining Spectroscopic and Photometric Surveys
}

\author{
Enrique Gaztañaga* \\ Instituto de Ciencias del Espacio \\ E-mail: gaztanaga@gmail.com
}

\begin{abstract}
The search for the nature of the dark sector relies on the combination of multiple techniques and probes, from both spectroscopic and photometric data. This matches well with the fact that some probes are intrinsically 3D (like RSD) and some 2D (like WL). But to get the best constraints we need to combine all of these. We show how using angular cross-correlations we can recover the full 3D galaxy clustering information, including BAO and RSD in spectroscopic surveys. This allows the combination of spectroscopic and photometric galaxy surveys, including photo-z error calibration and addition of WL. We show some application of these ideas in current data and simulations and show how overlapping surveys result in both better constrains and better understanding of systematic errors.
\end{abstract}

Frontiers of Fundamental Physics 14 - FFP14,

15-18 July 2014

Aix Marseille University (AMU) Saint-Charles Campus, Marseille

\footnotetext{
${ }^{*}$ Speaker.
} 